\title{
Scanning X-ray Microscopy with a Single Photon Counting 2D Detector
}

\author{
K. StACHNiK ${ }^{a, *}$ AND A. MEENTS ${ }^{b}$ \\ ${ }^{a}$ AGH University of Science and Technology, Faculty of Physics and Applied Computer Science \\ Al. A. Mickiewicza 30, 30-059 Krakow, Poland \\ ${ }^{b}$ DESY Photon Science, Deutsches Elektronen-Synchrotron DESY, Notkestr. 85, 22607 Hamburg, Germany
}

The experimental application of a novel technique utilizing a high resolution 2D pixel detector (PILATUS) in scanning transmission X-ray microscopy is presented. Measurements were performed at beamline P11 at the PETRA III synchrotron light source. Results are compared to the approach based on a segmented-type detector. A full simulation of the experimental line has been implemented in $\mathrm{C}++$ programming language. Concepts of ptychography and its application in X-ray microscopy are also briefly discussed.

DOI: $10.12693 /$ APhysPolA.125.902

PACS: 41.50.+h, 87.59.-e, 68.37.Yz, 87.64.mh

\section{Introduction}

X-ray microscopy has been since a long time an important method for imaging of various types of specimen. With increasing performance of X-ray light sources and a significant development of computational power, in addition to a simple transmission approach, phase contrast $\mathrm{X}$-ray imaging has become an important technique. Main area of phase-sensitive methods are studies of low absorption specimen, such as biological samples. In this field, scanning transmission X-ray microscopy (STXM) may be considered to be a representative technique. An overview of X-ray diffraction microscopy, available techniques and their experimental realisations, can be found e.g. in $[1,2]$. In this paper we present an experimental application of STXM based on differential phase contrast (DPC). So far, reconstruction methods such as DPC have usually required dedicated segmented detectors [3] because fully pixelated 2D detectors, such as charged coupled devices $(\mathrm{CCDs})$, were rather slow and noisy. However, due to a significant progress in detector development, 2D arrays of single photon counters have become available recently. Such a pixel detector was used for the measurements presented in this paper.

\section{Differential phase contrast}

A scanning microscope provides two major types of contrast mechanisms: absorption contrast and differential contrast. While the former is a function of specimen's thickness, the latter depends on the thickness gradient.

A typical experimental setup used for STXM measurements is shown in Fig. 1. It enables the evaluation of both types of contrasts mentioned above. An incident beam is

*corresponding author; e-mail: karolina.stachnik@gmail.com

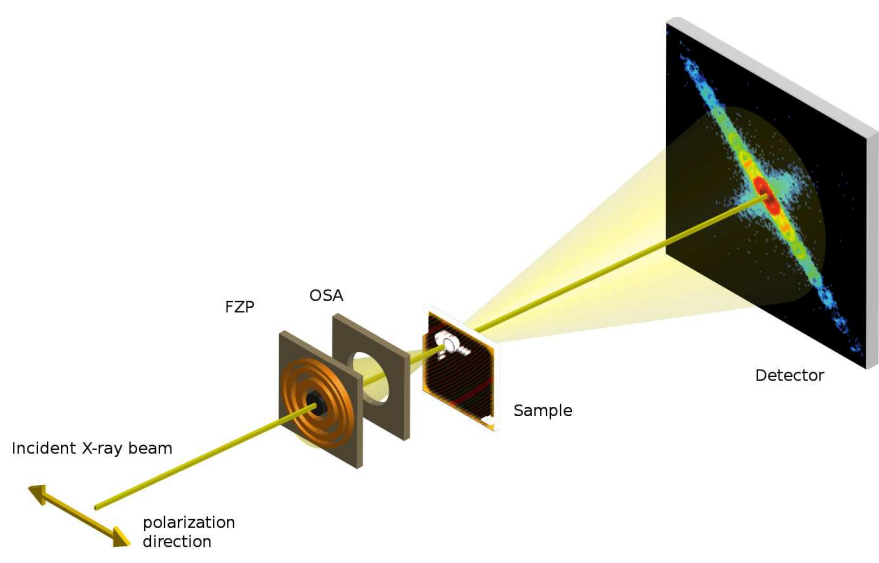

Fig. 1. Schematic view of the experimental setup used for STXM measurements.

focused down to a small spot by a Fresnel zone plate and the first order focus is selected using an order sorting aperture (OSA). The sample is raster-scanned through the focused beam. For each scan point the transmitted distribution of far-field intensity is measured by an area sensitive detector. If we consider a sample characterized by a lateral thickness function $\Delta z(\boldsymbol{r})$ and a complex index of refraction, $n=1-\delta-\mathrm{i} \beta$, the object transmission function $O(\boldsymbol{r})$ is defined as:

$$
O(\boldsymbol{r})=\exp (+\mathrm{i} \delta k \Delta z) \exp (-\beta k \Delta z)
$$

where $k=2 \pi / \lambda$ is the wave number, and $\delta$ and $\beta$ are refractive index decrement and absorption index, respectively. An incident wave field is described by the illumination function $P(\boldsymbol{r})$ and called a probe. Having interacted with the sample, it propagates over a distance $d$ to the detector plane $\boldsymbol{r}^{\prime}$. The intensity measured by the scanning microscope is proportional to the squared modulus of a convolution of the object and probe functions 


$$
I\left(\boldsymbol{\omega}^{\prime} ; \boldsymbol{R}_{s}\right) \propto\left|O\left(\boldsymbol{\omega}^{\prime}\right) \otimes\left(P\left(\boldsymbol{\omega}^{\prime}\right) \mathrm{e}^{\mathrm{i} \boldsymbol{R}_{s} \boldsymbol{\omega}^{\prime}}\right)\right|^{2},
$$

where spatial frequency is expressed as $\boldsymbol{\omega}^{\prime}=(k / d) \boldsymbol{r}^{\prime}$ and $\boldsymbol{R}_{s}$ defines a displacement of the probe with respect to the specimen in a plane perpendicular to the beam axis. In phase contrast imaging, once the beam passes through the sample, small phase shifts in different parts of the beam are converted into intensity contrast in a final image. When refracted, X-rays undergo a considerably small angular deviation, though it can be accurately measured. From a variety of methods, we have chosen the differential phase contrast technique to determine such deviation and hence to obtain the phase gradient image of a sample.

We can consider the first order approximation of the object function exponent around the point illuminated by the probe. For simplicity, we set the origin of coordinates in this point and, from now on, refer to it by the index 0 . Then, the general expression for the intensity in the detector plane of a scanning microscope is given [1] by

$$
\begin{gathered}
I\left(\boldsymbol{r}^{\prime} ; \boldsymbol{R}_{s}\right)=\exp \left(-\left.2(\beta k \Delta z)\right|_{0}\right) \\
I_{O(\boldsymbol{r})=1}\left(\boldsymbol{r}^{\prime}+\left.\frac{d}{k} \nabla_{\perp}(\delta k \Delta z)\right|_{0}\right) .
\end{gathered}
$$

Therefore, it can be identified as the intensity in the absence of specimen $I_{O(\boldsymbol{r})=1}\left(\boldsymbol{r}^{\prime}\right)$ shifted by an amount proportional to the phase gradient, $\left.(d / k) \nabla(\delta k \Delta z)\right|_{0}$, and attenuated by the specimen absorption, $\exp \left(-\left.2(\beta k \Delta z)\right|_{0}\right)$. This means that measured intensity is no longer a symmetric function with respect to the origin and its centre of mass and centre of symmetry are shifted. This motivates definitions of two approaches for calculating differential phase contrast discussed within the scope of this work. The first approach is based on a quadrant detector which consists of four equal square segments. Before measurement it should be symmetrically aligned so that the direct beam position points at a common vertex of all segments.

After introducing the specimen, angular deviation of the beam causes the intensity distribution to shift in horizontal and vertical directions. The differential phase contrast signals, $\mathrm{DPC}_{x}$ and $\mathrm{DPC}_{y}$ respectively, can provide the way to quantify such deflections. Their complete definition for the quadrant detector approach is as follows:

$$
\begin{aligned}
\mathrm{DPC}_{x} & =\frac{I_{\mathrm{R}}-I_{\mathrm{L}}}{I_{\text {total }}}, \\
\mathrm{DPC}_{y} & =\frac{I_{\mathrm{B}}-I_{\mathrm{T}}}{I_{\text {total }}},
\end{aligned}
$$

where subscripts denote integrated intensities from respectively: $\mathrm{R}$ - right, $\mathrm{L}$ - left, $\mathrm{B}$ - bottom, and $\mathrm{T}-$ top segments of a quadrant detector and $I_{\text {total }}$ is a total integrated intensity measured for a particular scan point.

The second approach of evaluating DPC signals is based on determining a centre of mass of a diffracted intensity distribution. Such approach can be utilized experimentally once a $2 \mathrm{D}$ pixel detector is available. If we consider a scan consisting of $N$ recorded intensity distributions $I_{j}, j=1, \ldots, N$, and if $\boldsymbol{r}^{\prime}=\left(x^{\prime}, y^{\prime}\right)$ is a real space coordinate system of detector pixels, then the differential phase contrast signals can be given by

$$
\begin{aligned}
\operatorname{DPC}_{x}^{j} & =\frac{\sum_{\boldsymbol{r}^{\prime}} x^{\prime} I_{j}\left(\boldsymbol{r}^{\prime}\right)}{\sum_{\boldsymbol{r}^{\prime}} I_{j}\left(\boldsymbol{r}^{\prime}\right)}-x_{\mathrm{ref}}^{\prime}, \\
\mathrm{DPC}_{y}^{j} & =\frac{\sum_{\boldsymbol{r}^{\prime}} y^{\prime} I_{j}\left(\boldsymbol{r}^{\prime}\right)}{\sum_{\boldsymbol{r}^{\prime}} I_{j}\left(\boldsymbol{r}^{\prime}\right)}-y_{\mathrm{ref}}^{\prime},
\end{aligned}
$$

where the direct beam points at a reference point $\left(x_{\mathrm{ref}}^{\prime}, y_{\mathrm{ref}}^{\prime}\right)$. Implementation of this approach in the case of single photon counting 2D detector allows to use, by default, all valid pixels. Yet, both approaches of calculating DPC signals enable the application of specific masks to select desired regions of interest.

\section{Experimental setup}

The measurements were performed at beamline P11 at the PETRA III light source, DESY, Hamburg. A schematic view of the experimental setup is shown in Fig. 1. The photon energy was $6.2 \mathrm{keV}$. We used a focusing Fresnel zone plate (FZP) of $100 \mu \mathrm{m}$ diameter and $25 \mathrm{~nm}$ outermost zone width. Upstream to the FZP, a central stop of $50 \mu \mathrm{m}$ diameter was placed to prevent a direct beam from hitting the detector. To isolate the first order focus, an order sorting aperture of $10 \mu \mathrm{m}$ diameter was placed downstream to the FZP. The scanned object was positioned in the back focal plane at the distance of $12.5 \mathrm{~mm}$ downstream to the FZP on a computer-controlled piezomotorized $x / y$ stage. The sample-detector distance of $3.95 \mathrm{~m}$ was bridged by a vacuum flight tube. To record diffraction patterns we used the 2D pixel detector PILATUS 6M [4, 5], developed at the Paul Scherrer Institute, Switzerland. The PILATUS $6 \mathrm{M}$ detector is subdivided into $5 \times 12$ modules. Every module consists of approximately 100,000 pixels, each of size $172 \times 172 \mu \mathrm{m}^{2}$, and is operating in single photon counting mode. The acquisition time per projection was set to $1 \mathrm{~s}$. The sample was raster-scanned with step sizes of $250 \mathrm{~nm}$. The positioning accuracy was better than $25 \mathrm{~nm}$. As a test sample we used an absorbing Siemens star of $20 \mu \mathrm{m}$ diameter and with 18 spokes.

\section{Simulation}

For testing parameters of the experimental setup as well as evaluating data, a numerical simulation tool has been developed. Its major aim is to propagate an X-ray wavefield through elements of the experimental setup in order to generate a complete dataset. The software has been implemented in $\mathrm{C}++$ programming language with data analysis and image processing supported by a $\mathrm{C}++$ based ROOT Data Analysis Framework [6]. The created library consists of classes operating on real and complex two-dimensional arrays, performing wavefield propagation, simulating various optical elements such as: FZP, central stop (CS), pinhole (e.g. order sorting aperture, 
OSA), and a class handling a test sample e.g. a Siemens star. Test samples can be also loaded in graphic file formats. The simulation software is designed in a way enabling users to build their own experimental setup, thus various configurations can be tested. The wavefield propagation required a careful implementation of propagator functions. Within the paraxial approximation (meaning that the angle between wave vector and direction of propagation is small), the numerical implementation of the propagation of a complex two-dimensional wavefield $E_{\omega}(x, y, z)$ between two planes, $z=z_{1}$ and $z=z_{2}$, can be done in two ways [1]. The first is a convolutional approach, often referred to as an angular spectrum:

$$
\begin{aligned}
& E_{\omega}\left(x_{2}, y_{2}, z_{2}\right)=\mathrm{e}^{\mathrm{i} k \Delta z} \\
& \quad \mathcal{F}^{-1}\left(\mathrm{e}^{-\frac{\mathrm{i} \Delta z}{2 k}\left(k_{x}^{2}+k_{y}^{2}\right)} \mathcal{F}\left(E_{\omega}\left(x_{1}, y_{1}, z_{1}\right)\right)\right),
\end{aligned}
$$

where $\mathcal{F}$ denotes the $2 \mathrm{D}$ Fourier transform $(\mathrm{FT})$ and $\Delta z=z_{2}-z_{1}$. The second is derived from a direct application of the Fresnel diffraction integral and can be realized by the following implementation:

$$
\begin{gathered}
E_{\omega}\left(x_{2}, y_{2}, z_{2}\right)=\mathrm{e}^{\mathrm{i} k \Delta z} P_{\Delta z}\left(x_{2}, y_{2}\right) \\
\mathcal{F}\left(E_{\omega}\left(x_{1}, y_{1}, z_{1}\right) P_{\Delta z}\left(x_{1}, y_{1}\right)\right),
\end{gathered}
$$

where $P_{\Delta z}(x, y)=\exp \left(\frac{\mathrm{i} k}{2 \Delta z}\left(x^{2}+y^{2}\right)\right)$ is a phase factor in a real space. Mathematically, these two formulae are equivalent and can be used in both near-field and far-field regimes. However, due to $\Delta z$ appearing in the denominator of the phase factor $P_{\Delta z}(x, y)$, the formula in (9) is numerically more suitable for propagation at large distances. In particular, at sufficiently large distance, $P_{\Delta z}(x, y)$ approaches one and can be neglected, and then formula (9) takes the form relevant to the Fraunhofer diffraction. The Fraunhofer approximation is fulfilled if the Fresnel number defined as $N_{\mathrm{F}}=D^{2} /(\lambda \Delta z) \ll 1$, where $D$ is a characteristic distance in the plane at $z_{1}$. In numerical calculations a discrete $2 \mathrm{D}$ Fourier transform [7], implemented in a FFTW fast Fourier transform package [8], has been used. If $M\left(x_{0}, y_{0}\right)$ is a $N_{x} \times N_{y}$ matrix representing an initial (complex) field, then the discrete 2D Fourier transform is defined as:

$$
\begin{aligned}
& M(x, y)=\frac{1}{\sqrt{N_{x} N_{y}}} \\
& \times \sum_{x_{0}=0}^{N_{x}-1} \sum_{y_{0}=0}^{N_{y}-1} M\left(x_{0}, y_{0}\right) \mathrm{e}^{-\mathrm{i}\left(x x_{0} / N_{x}+y y_{0} / N_{y}\right)} .
\end{aligned}
$$

It should be noted that the convolutional approach preserves the original pixel size of a discrete two-dimensional field as a result of applying both forward and inverse FT. On the other hand, due to applying only a single FT, the approach in (9) involves a change of the pixel size. If we consider a discrete, two-dimensional input wavefield of $N_{x} \times N_{y}$ pixels, with pixel sizes equal to $\Delta x_{1}$ and $\Delta y_{1}$, then, after propagation over a distance $\Delta z=z_{2}-z_{1}$, pixel sizes of an output wavefield are determined by the Fourier transform properties [7] and given by

$$
\Delta p_{z_{2}}=\frac{\lambda \Delta z}{N_{p} \Delta p_{z_{1}}}, \quad \text { where } p=x, y .
$$

In all propagations where closely positioned optical elements of the experimental line up to the sample, the approach based on (8) is used. Only in the case of propagation to the detector, the far field approach based on (9) with $P_{\Delta z}(x, y)=1$ is used. As an example for the application of the simulation software, the propagation of X-rays $(\lambda=0.2 \mathrm{~nm})$ through a simple absorbing Siemens star of $20 \mu \mathrm{m}$ diameter has been calculated. The initial intensity of each pixel has been assumed to be unity. The results are shown in Fig. 2, where intensity distributions of the initial and the final wavefields in near-field $(\Delta z=4 \mathrm{~mm})$ and in far-field $(\Delta z=5.504 \mathrm{~m})$ regimes are plotted.

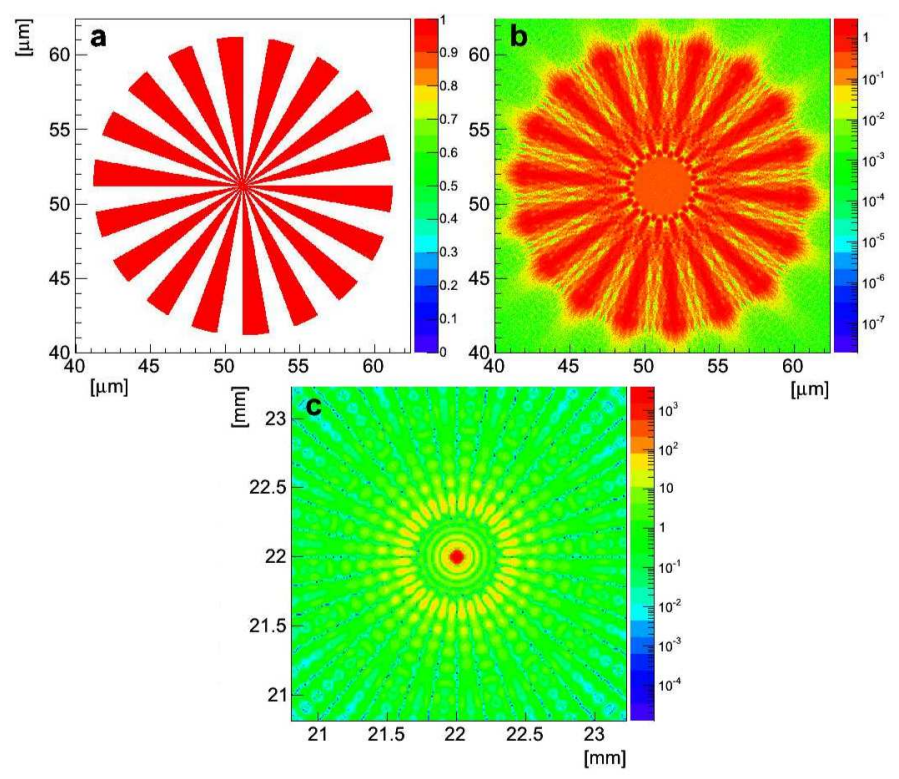

Fig. 2. Simulated intensity distributions of (a) initial wave field, and of the wave fields propagated to (b) $\Delta z=$ $4 \mathrm{~mm}$ and (c) $\Delta z=5.504 \mathrm{~m}$.

\section{Results}

In this section we present results of DPC reconstructions of both the simulated object and of the real measurement at beamline P11 of PETRA III light source. The test object in both cases was an absorbing Siemens star of $20 \mu \mathrm{m}$ diameter and 18 spokes. Details of the experimental setup are described in Sect. 3. For simulation purposes, due to memory restriction, we used a focusing Fresnel zone plate of $50 \mu \mathrm{m}$ diameter and $125 \mathrm{~nm}$ outermost zone width. In both cases the object (Siemens star) was situated in the back focal plane and raster-scanned with $250 \mathrm{~nm}$ step size.

Results of the simulated scan are shown in Fig. 3. Horizontal and vertical components of DPC are reconstructed directly from pixels. One can see that the shape of the Siemens star is well reproduced. 


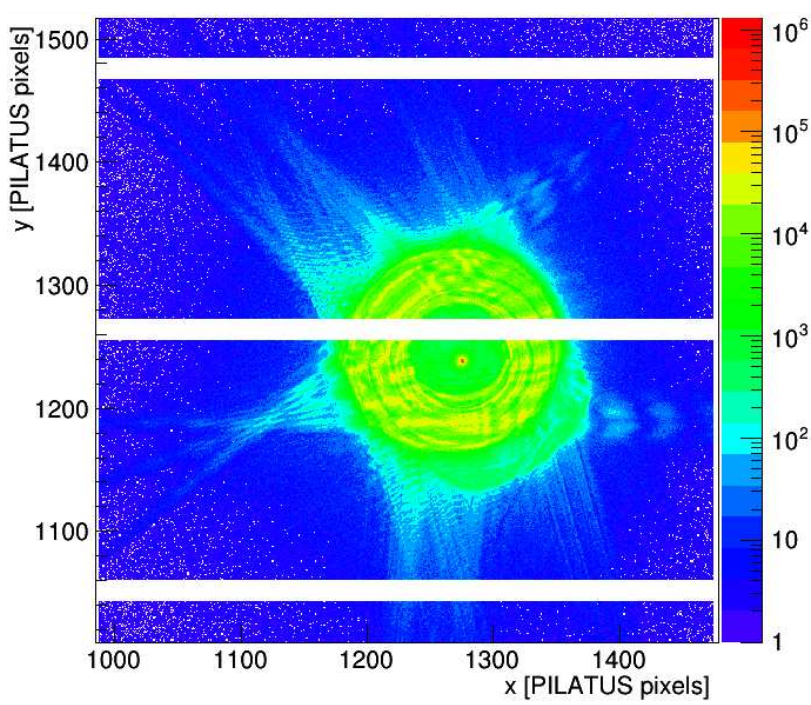

Fig. 3. An example of diffraction patterns recorded by the PILATUS detector during the scan of a Siemens star.

In Fig. 4 we present a typical diffraction pattern recorded by the PILATUS detector. The pattern is affected by the inactive gaps between adjacent modules of the detector. It is also clearly seen from asymmetry in the central part of the image in Fig. 4 that the central stop and the Fresnel zone plate were not precisely aligned.

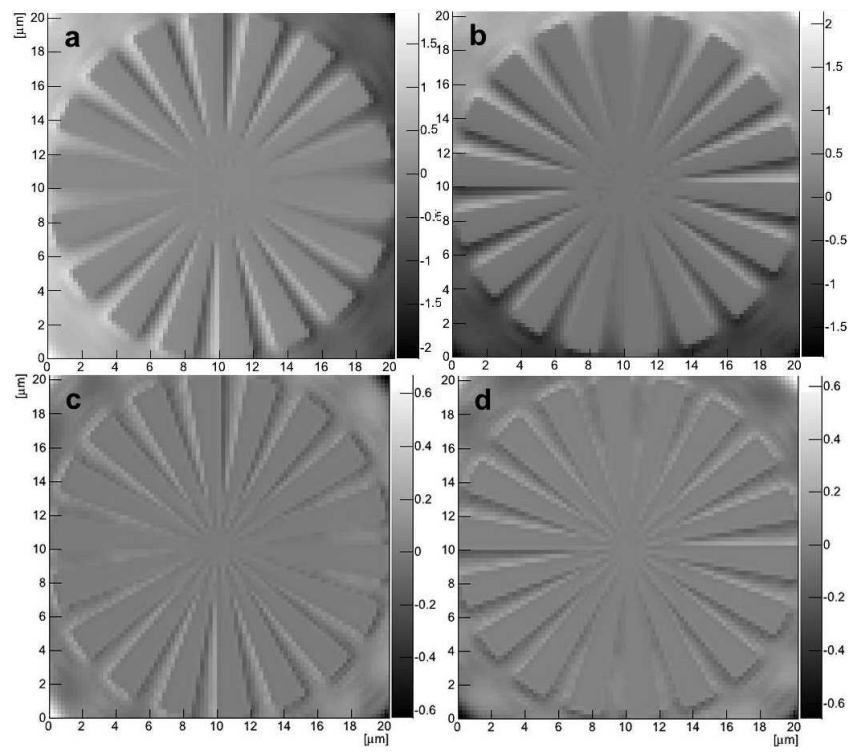

Fig. 4. Differential phase contrast analysis of simulated data: (a), (b) $\mathrm{DPC}_{x}$ and $\mathrm{DPC}_{y}$ signals calculated from the pixel approach; (c), (d) $\mathrm{DPC}_{x}$ and $\mathrm{DPC}_{y}$ signals based on the quadrant detector approach.

In Fig. 5 we present DPC reconstruction from real data. The center of the diffraction pattern was assumed to be a pixel of the highest signal lying on the direction defined by the direct beam (beam spot). Two approaches
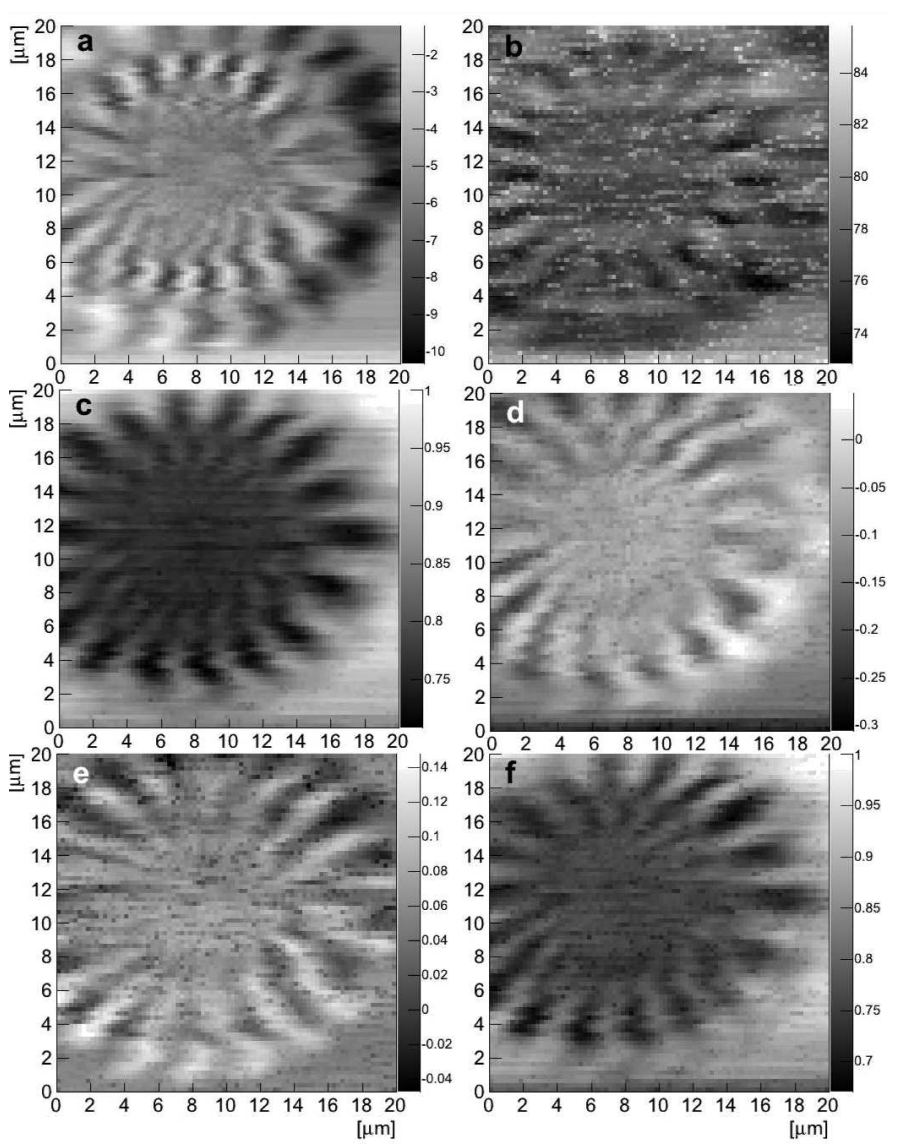

Fig. 5. Differential phase contrast analysis of real data: (a)-(c) pixel approach, (d)-(e) quadrant detector approach. $\mathrm{DPC}_{x}$ signals in: (a), (d), DPC $y$ signals in: (b), (e), and transmission in: (c), (f).

have been used for the DPC reconstruction: direct calculation from PILATUS pixels (Fig. 5a,b) and an approach based on the quadrant detector (emulated from PILATUS data, Fig. 5d,e). Images in Fig. 5a,d and Fig. 5b,e depict $\mathrm{DPC}_{x}$ and $\mathrm{DPC}_{y}$ signals, respectively, whereas those in Fig. 5 c,f present transmission signal. The quality of reconstruction in both approaches is poor. This is partly due to the misalignment of the central stop, but mostly because of inaccurate operation of the scanning system during each projection. The problem with probe positioning must be solved before moving to our main objective which is to establish at the P11 beamline a measurement setup for ptychographic reconstruction. The general idea of ptychography is explained briefly in the next section.

\section{Ptychography}

With an increasing brilliant performance of synchrotron light sources and a significant hardware development it has become feasible to combine prominent features of coherent diffractive imaging with scanning miscroscopy. As a result, a novel method, called ptychogra- 
phy, has evolved, establishing a new standard in modern high resolution X-ray imaging. Ptychography (from the Greek word " $\pi \tau v \xi$ " meaning "to fold") was originally developed in electron microscopy $[9,10]$ in 1970 s.

If prerequisites for a complete inversion of a scattering problem, such as high degree of coherence, high signal-to-noise ratio and oversampling, are fulfilled, then ptychography can be utilized also for X-rays. An experimental setup can be organized similarly to a STXM. Yet, unlike in the conventional STXM, ptychography uses coherent $\mathrm{X}$-ray radiation in such way that the transmitted signal is spatially resolved by an area detector rather than recorded just as an integrated signal. Thus, pixel array detectors such as single photon counters are favourable. In ptychography, diffraction orders, which come from the exit wave diffraction into the far-field, are folded (convoluted) with the Fourier transform of the illumination function in the object plane.

The phase problem in the reconstruction process can be ultimately resolved by observing how the intensity of overlapping parts of diffraction orders changes in successive projections as the sample is translated [11]. The information from these overlaps is obtained by setting a scan shift of the sample between subsequent projections below the extent of the localized illumination (the probe). Resolution in ptychographical imaging is no longer limited by the size of the focal spot as in STXM. It is significantly improved due to application of coherent radiation and area pixel detector and determined by basic parameters of an experiment according to the formula (11). A complete ptychographical dataset, which consists of recorded intensity distributions and illumination function positions, allows to regain a sample image. The strategy is to iteratively reconstruct and refine single projection of the sample which is consistent with all collected diffraction patterns [12]. From a variety of reconstruction algorithms we have chosen to develop our proprietary software based on the extended ptychographical iterative engine [13] which allows to regain both the probe and the object (a sample).

\section{Summary}

In preparation for establishing a setup for ptychographical measurements of biological samples at beamline P11 at the PETRA III light source, we have performed test experiment and simulation of a known object (Siemens star) using the DPC approach. A novel technique utilizing the pixel detector (PILATUS) for record- ing diffraction patterns was applied in parallel to the conventional technique, treating the PILATUS like a segmented detector. Although in DPC analysis both pixel and segmented detectors are equally suitable, in the ptychographical measurement the use of a pixel detector is essential. The quality of DPC reconstruction was substantially limited due to problems with precise positioning. These problems are now under thorough investigation. We have also prepared a standalone software package containing tools for simulation of the experimental line and image reconstruction which can be used for both simulated and real data. The code is written in $\mathrm{C}++$ programming language with the support of the $\mathrm{C}++$ based ROOT Data Analysis Framework.

\section{References}

[1] J. Als-Nielsen, D. McMorrow, Elements of Modern X-ray Physics, 2nd ed., Wiley, Chichester 2011.

[2] P. Thibault, V. Elser, Annu. Rev. Condens. Matter Phys. 1, 237 (2010).

[3] M. Feser, B. Hornberger, C. Jacobsen, G. De Geronimo, P. Rehak, P. Holl, L. Strüder, Nucl. Instrum. Methods Phys. Res. A 565, 841 (2006).

[4] B. Henrich, A. Bergamaschi, C. Broennimann, R. Dinapoli, E.F. Eikenberry, I. Johnson, M. Kobas, P. Kraft, A. Mozzanica, B. Schmitt, Nucl. Instrum. Methods Phys. Res. A 607, 247 (2009).

[5] P. Kraft, A. Bergamaschi, Ch. Broennimann, R. Dinapoli, E.F. Eikenberry, B. Henrich, I. Johnson, A. Mozzanica, C.M. Schlepütz, P.R. Willmott, B. Schmitt, J. Synchrotron Rad. 16, 368 (2009).

[6] R. Brun, F. Rademakers, Nucl. Instrum. Methods Phys. Res. A 389, 81 (1997). See also http://root. cern.ch/.

[7] O.K. Ersoy, Diffraction, Fourier Optics and Imaging, Wiley, New Jersey 2007.

[8] M. Frigo, S.G. Johnson, Proc. IEEE 93, 216 (2005).

[9] W. Hoppe, Acta Crystallogr. A 25, 508 (1969).

[10] R. Hegerl, W. Hoppe, Ber. Bunsen-Ges. Phys. Chem. 74, 1148 (1970).

[11] J.M. Rodenburg, Adv. Imaging Electron Phys. 150, 87 (2008).

[12] M. Dierolf, O. Bunk, S. Kynde, P. Thibault, I. Johnson, A. Menzel, K. Jefimovs, Ch. David, O. Marti, F. Pfeiffer, Europhys. News 39, 22 (2008).

[13] A.M. Maiden, J.M. Rodenburg, Ultramicroscopy 109, 1256 (2009). 